\title{
Fragilidades no cuidado em saúde mental: percepções de profissionais da Atenção Primária à Saúde em tempos de COVID-19
}

\author{
Weaknesses in mental health care: perceptions of primary health care professionals in times of
} COVID-19

Debilidades en la atención de salud mental: percepciones de los profesionales de atención primaria en la época del COVID-19

\section{Resumo}

Objetivo: analisar a percepção dos profissionais de saúde da Atenção Primária à Saúde, acerca das fragilidades relacionadas ao cuidado em saúde mental em seu cotidiano de trabalho, e identificar propostas para qualificar a assistência a esta demanda, em meio à pandemia ocasionada pela COVID-19. Método: pesquisa-ação, desenvolvida em um município ao norte do Rio Grande do Sul, Brasil, cujos participantes foram profissionais de uma Unidade Básica de Saúde. As informações foram produzidas em dezembro de 2020, por meio de roda de conversa. O tratamento dos dados foi guiado pela análise de conteúdo. Resultados: As fragilidades no cuidado envolveram principalmente, o tratamento a base de psicotrópicos, a assistência inadequada às crianças e adolescentes em vulnerabilidade social no período da pandemia por COVID-19 e aos usuários em sofrimento mental e suas famílias. A educação permanente, o desenvolvimento de ações de saúde mental à população e o cuidado integral, emergiram como propostas para qualificar a assistência. Conclusão: identificou-se ser necessária uma reestruturação urgente do cuidado em saúde mental na Atenção Primária à Saúde, principalmente devido ao risco a que estão expostas algumas pessoas, com o uso inadequado de psicotrópicos. Compreende-se que o enfermeiro, ao possuir competências adequadas (conhecimentos, habilidades e atitudes), pode através de seu conhecimento e atuação nas equipes de ESF, contribuir para a qualificação da assistência em saúde mental na APS.

Palavras-chave: Atenção primária à saúde; Saúde mental; Profissionais de saúde; Assistência à saúde mental; COVID-19.

\footnotetext{
Abstract

Aim: to analyze the perception of health professionals in Primary Health Care about the weaknesses related to mental health care in their daily work, and identify proposals to qualify assistance to this demand, amidst the pandemic caused by COVID-19. Method: it is an action-research, developed in a municipality in the north of Rio Grande do Sul, Brazil, whose participants were 8 professionals from a Basic Health Unit. The information was produced in December 2020, through a conversation circle. Data treatment was guided through content analysis. Results: the weaknesses in care involved the centralization of treatment based on psychotropic drugs, inadequate care for children and adolescents in social vulnerability, as well as users in mental suffering and their families during the COVID-19 pandemic period. Continuing education, the development of mental health actions to the population and comprehensive care emerged as proposals to qualify care. Conclusion: it was identified that a restructuring of mental health care in Primary Health Care is necessary since the prescription of psychotropic drugs is the first and
} 
only treatment option for users. It is understood that nurses, on having adequate competencies (knowledge, habilities and attitudes), can contribute to the qualification of mental health care in Primary Health Care.

Keywords: Primary health care; Mental health; Health professionals; Mental health care; COVID-19.

\section{Resumen}

Objetivo: analizar la percepción de los profesionales de la salud en Atención Primaria de Salud sobre las debilidades relacionadas con la atención en salud mental en su quehacer diario, e identificar propuestas para calificar la atención a esta demanda, en medio de la pandemia provocada por el COVID-19. Método: se trata de una investigación acción, desarrollada en una ciudad al norte de Rio Grande do Sul, Brasil, cuyos participantes fueron 8 profesionales de una Unidad Básica de Salud, la información fue producida en diciembre de 2020, a través de una rueda de conversación. El tratamiento de los datos se guió por el análisis de contenido. Resultados: las debilidades en la atención involucraron la centralización del tratamiento a base de psicofármacos, la atención inadecuada a los niños y adolescentes en vulnerabilidad social, así como a los usuarios en sufrimiento mental y sus familias durante el período pandémico del COVID-19. Educación continua, el desarrollo de Las acciones de salud mental a la población y la atención integral surgieron como propuestas para calificar la asistencia. Conclusión: se identificó que es necesaria una reestructuración de la atención en salud mental en la Atención Primaria de Salud, debido a que la prescripción de psicofármacos es la primera y única opción de tratamiento para los usuarios. Se entiende que el enfermero al contar con las competencias adecuadas (conocimiento, habilidades y actitudes), puede através de su conocimiento y desempeño em los equipos de la Estrategia Salud de la Familia, contribuir a la calificación de la atención en salud mental en Atención Primaria de Salud.

Palabras clave: Primeros auxilios; Salud mental; Profesionales de la salud; Cuidado de la salud mental; COVID-19.

\section{Introdução}

O cuidado adequado em saúde mental é um tema que vem sendo discutido no Brasil desde o início da Reforma Psiquiátrica, sendo priorizada uma assistência integral com enfoque não apenas aos indivíduos em sofrimento mental e às suas famílias, mas também no desenvolvimento de ações de promoção e prevenção aos agravos de saúde mental na população (Barros \& et al., 2019).

Atualmente, a literatura científica recomenda que sejam utilizadas tecnologias leves, como o acolhimento, a escuta qualificada, as visitas domiciliares, os grupos, as Práticas Integrativas e Complementares (PICs) e a ampliação da clínica, no cuidado em saúde mental na Atenção Primária à Saúde (APS), pois as mesmas, abarcam a dimensão subjetiva dos indivíduos bem como, as situações a serem enfrentadas (Ávila \& Siniak, 2017; Campos, Bezerra \& Jorge, 2018; Franco \& Merhy, 2012; Brasil, 2006). Porém, o que se observa na maioria das Unidades Básicas de Saúde (UBS), é a realização de um cuidado fragmentado, curativo, com predomínio da medicalização como assistência às pessoas em sofrimento mental e raras ações de promoção e prevenção destes agravos na população, ocasionando uma assistência pouco resolutiva. Pode-se afirmar que pensar na saúde mental de forma fragmentada pode resultar em ações que não beneficiarão o sujeito, sua família, rede social e comunidade (Dias \& et al., 2020).

No ano de 2020, a pandemia da Coronavirus Disease 2019 (COVID-19), emergiu como um grave problema de saúde pública global que se espalhou rapidamente, partindo da China para outras partes do mundo (Nishiura, 2020). Durante períodos pandêmicos, tais como o que a população mundial vive atualmente, ocorre um aumento na prevalência de sintomas de ansiedade, estresse e transtornos mentais entre as pessoas. Além disso, a pandemia da COVID-19 implica outras esferas da vida, impondo mudanças nos modos de viver, trabalhar e se organizar, o que leva a sentimentos de desamparo e abandono e ao aumento da insegurança com relação ao futuro (Ornell \& et al., 2020).

Frente a este contexto, a literatura pontua ser de extrema importância o papel das equipes da APS no cuidado à saúde mental da população durante a pandemia da COVID-19. Isso se deve, principalmente, à proximidade e vínculo existentes entre os profissionais e as pessoas do território. Tal fato, possibilita a identificação de indivíduos com maior risco para o adoecimento mental relacionado à pandemia. Além disso, permite a articulação intersetorial, que pode auxiliar nas necessidades das famílias em maior vulnerabilidade, oferecendo orientações confiáveis às pessoas, voltadas a minimizar o sofrimento mental durante o isolamento e a importância da manutenção de uma rotina diária, que respeite o tempo de sono e 
horários das refeições, bem como, a inclusão de atividades físicas e de lazer, da interação com familiares e amigos, mesmo que à distância, e por fim, o apoio para minimizar a vivência do luto daqueles que perderam entes queridos (Nabuco, 2020; Martini, 2020).

Ao refletir sobre este fenômeno, este estudo teve como objetivo analisar a percepção dos profissionais de saúde da APS, acerca das fragilidades relacionadas ao cuidado em saúde mental em seu cotidiano de trabalho e identificar propostas para qualificar a assistência a esta demanda, em meio à pandemia ocasionada pela COVID-19.

\section{Metodologia}

Trata-se de uma pesquisa-ação adaptada de Thiollent (2011). Neste método os atores implicados participam na resolução dos problemas, com conhecimentos diferenciados, propondo soluções e aprendendo na ação. Os pesquisadores exercem um papel articulador e facilitador em contato com os interessados (Thiollent, 2011). Neste estudo foi realizada uma roda de conversa, com profissionais da equipe multiprofissional de uma UBS, de um município de pequeno porte, localizado ao norte do Rio Grande do Sul. As rodas de conversas são espaços coletivos usados para a discussão e reflexão sobre diversos temas, podendo ser utilizadas para distintos fins, inclusive para o planejamento de ações (Machado, Carvalho, Brandão \& Vilarinho, 2015).

Os critérios de inclusão dos participantes foram definidos previamente, sendo eles: ser maior de 18 anos, ser profissional de nível superior ou médio, atuar como médicos, enfermeiros, técnicos de enfermagem e psicóloga da APS, atuar no mínimo há seis meses na APS. Os critérios de exclusão foram: estar afastado de suas atividades laborais por motivo de doença ou ser demitido de suas funções. Optou-se em restringir a pesquisa a estes profissionais da APS do município, uma vez que cada um desempenha funções específicas na prática, porém a demanda de usuários é a mesma para todos, e, portanto, entende-se que para a aplicabilidade do constructo a equipe deve responsabilizar-se de forma coletiva (Brasil, 2006).

Cabe ressaltar que os resultados deste artigo fazem parte da terceira etapa da pesquisa-ação desenvolvida na dissertação intitulada: Desenvolvimento de Cartilha Educativa sobre o Manejo de Usuários em Sofrimento Mental na Atenção Primária à Saúde. Na pesquisa-ação foram percorridas as seguintes etapas: 1) Fase exploratória: Revisão Integrativa e identificação do perfil de usuários de psicotrópicos; 2) Campo de observação e representatividade qualitativa: identificação das potencialidades e fragilidades do atendimento prestado aos usuários em sofrimento mental, principais Transtornos Mentais atendidos e situações em saúde mental de difícil manejo; 3) Coleta de dados: entrevista individual e roda de conversa com equipe multiprofissional; 4) Aprendizagem, saber formal/informal: planejamento, elaboração e validação da cartilha educativa; 5) Divulgação externa: roda de conversa para avaliação da cartilha educativa pelos profissionais; publicação de artigos científicos e apresentação do estudo em eventos científicos.

A produção das informações desta etapa do estudo ocorreu no mês de dezembro de 2020, por meio de uma roda de conversa, que durou aproximadamente 1 hora. Participaram da atividade três médicos, duas enfermeiras, uma psicóloga e dois técnicos de enfermagem. A roda de conversa foi realizada de modo presencial na UBS, em horário e data combinados previamente. Foram respeitados os protocolos de segurança para proteção e prevenção da Covid-19.

No primeiro momento, realizou-se o acolhimento dos participantes, sendo que na sequência, foram apresentados os dados obtidos do perfil de usuários de psicotrópicos do município e das entrevistas individuais realizadas previamente, com estes profissionais, sobre saúde mental. Foram utilizadas as seguintes questões disparadoras para mediar o debate acerca da temática com os participantes, de modo a obter a visão do grupo sobre o tema: na opinião de vocês quais os fatores que podem estar relacionados ao elevado consumo de psicotrópicos pelos usuários? Na opinião de vocês, como têm se dado a assistência em saúde mental na UBS? Que estratégias poderiam ser implantadas para qualificar o cuidado em saúde mental da população 
local? A atividade foi áudio-gravada e as gravações foram transcritas para posterior análise. O perfil dos profissionais participantes da pesquisa foi apresentado em números absolutos e porcentagens.

Para análise dos dados utilizou-se a proposta de análise de conteúdo de Bardin (2011) a qual conta com três fases: préanálise, exploração do material e tratamento dos resultados, inferência e interpretação. A primeira fase (pré-análise) consistiu na organização dos materiais a serem analisados com o objetivo de torná-los operacionais, sistematizando as ideias iniciais. Já a exploração do material constituiu a segunda fase, e se deu por meio da exploração dos dados com a definição de categorias. A terceira etapa foi voltada ao tratamento dos resultados, inferência e interpretação, destinando-se à organização dos resultados; onde ocorreu a condensação e o destaque das informações para análise, culminando nas interpretações inferenciais. Com o objetivo de compreender os significados e sentidos dos discursos dos profissionais, foi realizada a leitura exaustiva do material transcrito, levando à constituição de dois núcleos de significados, chamados aqui de categorias temáticas.

A pesquisa atendeu à Resolução no 466/12 do Conselho Nacional de Saúde para pesquisas realizadas em seres humanos, foi submetida ao Comitê de Ética em Pesquisa da Universidade do Estado de Santa Catarina, e aprovada com o número de parecer $\mathrm{n}^{\circ}$ 4.407.279 em 18 de novembro de 2020 (Brasil, 2012). A identidade dos participantes foi preservada, sendo utilizadas as iniciais M (médico), E (enfermeira), T (técnico em enfermagem) e P (psicóloga), acompanhada de numeração, exemplo: M1, T2, E2 respectivamente. Os participantes assinaram o Termo de Consentimento Livre e Esclarecido (TCLE).

\section{Resultados e Discussão}

A maioria dos participantes era do sexo feminino ( $\mathrm{n}=7 ; 87,5 \%)$, com média de idade de 39 anos e média de tempo de atuação profissional de 10,5 anos. A seguir, serão apresentados os dados que compõem as categorias temáticas que emergiram durante análise dos dados: Práticas de cuidado às pessoas em sofrimento mental na Atenção Primária e Propostas para mudar a assistência em saúde mental.

\section{As práticas de cuidado às pessoas em sofrimento mental na Atenção Primária}

Esta categoria apresenta a visão dos participantes acerca do cuidado prestado às pessoas em sofrimento mental na APS. Destaca-se que o enfoque dos discursos permaneceu, predominantemente, em torno das fragilidades da assistência, principalmente envolvendo a questão do uso de psicotrópicos. A primeira fragilidade no cuidado em saúde mental mencionada pelos profissionais na APS, diz respeito a não aceitação, por parte dos usuários, de terapêuticas que não sejam medicamentosas, para tratar os sintomas de sofrimento mental, conforme observa-se no relato:

"Eu acho que não é só a questão de sair de graça (psicotrópico), mas se torna mais fácil, que daí ela não precisa se deslocar até a unidade, ou se deslocar para fazer os tratamentos, então eles sempre optam pelo mais fácil né" (P1).

Segundo os participantes, algumas pessoas que utilizam psicotrópicos, não necessariamente precisariam usá-los, porém acabam consumindo tais medicamentos por períodos longos, habituando-se aos efeitos dos mesmos, ocasionando uma "cronificação medicamentosa". "Uns tomam por costume, porque não é assim, é costume” (M1).

A prevalência do uso de psicotrópicos em pessoas com idade superior a 55 anos, especialmente em idosos, também emergiu a partir dos dados analisados. Os profissionais reconhecem os riscos envolvidos nesta prática, citando inclusive, exemplos de medicações que podem causar iatrogenias nesta população, conforme observa-se no relato: 
"Na verdade, tem uma lista que fala de todos medicamentos que são potencialmente nocivos para os idosos. No caso, a Amitriptilina está nesse meio, os benzodiazepínicos também. Então, o que acontece, quando tu prescreves Amitriptilina pros idosos, pode dar até um efeito rebote dessa medicação, piora mais a situação, geralmente, ele fica mais nervoso, mais agitado por causa disso, e é um risco potencial de queda porque eles ficam mais tontos, porque tanto a Amitriptilina quanto os benzodiazepínicos sedam de uma forma excessiva, é bem complicado isso" (M3).

A primeira autora desta pesquisa evidenciou em estudo realizado sobre o perfil de usuários de psicotrópicos do município, que algumas pessoas do território chegaram a utilizar, pelo menos, dois benzodiazepínicos diferentes em um período inferior a um ano. Ao apresentar estes dados aos profissionais participantes da roda de conversa, os mesmos atribuíram tal situação à falta de medicamento na farmácia da UBS, motivo que consequentemente, promove a troca de fármacos e, também, às consultas realizadas no Pronto Atendimento Municipal, o qual não dispõe de acesso aos prontuários eletrônicos das pessoas atendidas na UBS, o que acaba favorecendo a substituição de medicamentos pelo médico."Ah, não quer comprar, daí vai com o médico do Pronto Atendimento, que dá outro, ele não tem sistema, daí troca”. (E1) “Isso já aconteceu, de eu atender, usava um, ia lá trocava por outro, não tinha sistema, voltava aqui” (M3).

A partir da discussão na roda de conversa, foi possível identificar que as prescrições de psicotrópicos em alguns casos, ocorrem de maneira inadequada, expondo os usuários a possíveis agravos à saúde, devido a tal prática. "Na dúvida, tem médicos que prescrevem assim: que não olha o princípio ativo, vai no senso comum, dá o mais forte que tem” (T2). A utilização de dois ou mais psicofármacos pela mesma pessoa, também foi discutida pelo grupo, sendo pontuados os riscos envolvidos nesta prática, como as possíveis interações farmacológicas, conforme exemplifica-se no depoimento:

"Isso aconteceu quando eu entrei aqui, a gente não tinha muito índice para psiquiatra, não tinha muito paciente que ia para o psiquiatra, aí a gente mudou o modo de atender. Aí o psiquiatra me ligou, que um remédio estava potencializando o efeito de outro, então ela estava tendo crises, a partir do momento que ela começou usar os dois tipos de remédio juntos. Daí ela tirou, não deu nem 15/20 dias que ela parou, a paciente começou a ter melhora, que era por causa desta interação medicamentosa" (P1).

Outra questão discutida pelos profissionais foi a respeito de como se dá o consumo de psicotrópicos pelos usuários. Identificou-se que os participantes desconhecem a realidade envolvida neste contexto, demonstrando que inexiste um acompanhamento adequado a esta demanda. "Esses dados são das retiradas, agora como se dá a manipulação, o consumo destes medicamentos? Tomam? Não tomam? Tomam dois por vez?” (M2).

Os participantes relataram ter realizado uma investigação por parte da equipe, voltada a identificar os problemas que motivaram alguns usuários a buscarem constantemente atendimento na UBS, e concluíram que as principais causas envolvidas neste cenário, tinham origem em algum transtorno mental.

"Eu lembro que foi em 2018 que nós fizemos uma avaliação dos pacientes que vinham com muita frequência ao posto de saúde, uma lista enorme, e fomos visitar em equipe, e quase mais da metade, uns $75 \%$ tinham transtornos mentais, né. Se foi na casa dos pacientes, e sempre se encontrava alguma coisa, que a dor não era o problema, quer dizer não era físico, era dor emocional e sempre associado aos transtornos mentais” (M1).

A partir do relato do participante, pode-se atribuir tal cenário a dois fatores, o primeiro seria a existência de um ponto falho na assistência prestada aos usuários, uma vez que demonstra a incessante busca por atendimento, e o segundo ao elevado número de pessoas em sofrimento mental no município. A falta de busca ativa, ao encontro da coordenação e longitudinalidade na assistência, no que tange à saúde mental, também emergiram como aspectos que fragilizam o cuidado, sendo realizadas somente aos usuários com problemas graves, conforme exemplifica-se no depoimento: 
"Se for caso grave, vem no primeiro atendimento, é uma ideação suicida, não vem no segundo a gente faz uma busca ativa, pelas agentes de saúde, ou faz visita. Agora, se vem um caso leve, a gente não tem como buscar todos os casos que não vem, agora se é caso grave, e chegou como urgência a gente vai atrás" (P1).

A inexistência do acompanhamento à família dos usuários em sofrimento mental por parte da equipe foi percebida como uma fragilidade da assistência, apesar de ser considerada como importante e essencial para a resolutividade do agravo mental apresentado pelos indivíduos.

"Um grande desafio que eu percebo é o acompanhamento desses pacientes que estão em tratamento nas famílias. Por exemplo, a família tem um problema, a pessoa tem um problema, daí eles encaminham para internação, às vezes compulsória, às vezes voluntária. A pessoa vai, a gente acompanha o paciente lá, mas o impacto que essa internação teve na família, muitas vezes a gente não acompanha. Aí o paciente retorna, a família não tem estrutura nenhuma para seguir com esse acompanhamento, há trinta dias, quarenta dias, o paciente está com problema de novo, e aí agrava o problema" (E2).

Mesmo sendo indiscutível a importância da família no cuidado em saúde mental, os profissionais relataram que o abandono familiar é uma situação comumente encontrada na assistência aos usuários em sofrimento mental.

\begin{abstract}
"A gente teve vários casos que não tinha acompanhante para ir junto para a internação. Teve caso que a gente teve que disponibilizar xampu, ir atrás para comprar as coisas para internação, que não tinha familiar, aí nós fomos atrás de um amigo do cara para ir. Outra situação com o psiquiatra, não é obrigado, mas alguém tem que acompanhar, várias vezes foram técnicos (enfermagem) junto, porque a família não quer nem saber, então o problema, é muito mais profundo do que a gente percebe" (P1).
\end{abstract}

Também emergiu de forma marcante a preocupação dos profissionais acerca das consequências psicológicas que parecem acometer crianças e adolescentes que se encontram em situação de vulnerabilidade social, em virtude das medidas de prevenção impostas pela pandemia por COVID-19, bem como, pelo receio quanto aos desdobramentos da pandemia.

“Tudo aponta, os estudos dizem, que vão se agravar os índices de problema em saúde mental. Nós vamos enfrentar isso com crianças, adolescentes, hoje se tu pegares o impacto que está tendo esse distanciamento forçado para as crianças, de não estar indo na aula, de estar convivendo mais diretamente com as famílias, com os avós, isso a gente não tem noção da dimensão que isso vai dar" (E2).

"A gente tem aqui [município estudado] um grande número de vulnerabilidade social, às vezes, na escola, as crianças saíam daquele ambiente. Então pensa agora, só naquele ambiente, imagina quando vier à tona as consequências psicológicas" (P1).

\title{
Propostas para mudar a assistência em saúde mental
}

Esta categoria é composta pelas sugestões dos participantes acerca de estratégias que poderiam ser adotadas pelo serviço para qualificar a assistência em saúde mental. Optou-se neste estudo, por considerar as sugestões relacionadas às diversas ações e intervenções em saúde mental, pois compreende-se que a atuação dos profissionais da APS, deve ir além da assistência prestada às pessoas com diagnóstico de transtornos mentais ou em sofrimento mental, devendo contemplar também atividades de promoção e prevenção à saúde mental da população.

Dentre as sugestões citadas, estão a assistência com enfoque integral do usuário e sua família, a realização de processos de educação permanente sobre a temática para os profissionais, o desenvolvimento de ações voltadas à população sobre as questões de saúde mental, a implantação de espaços de lazer e atividade física no município e o desenvolvimento de ações de prevenção e promoção à saúde mental para crianças. 
"Eu acho que a maioria dos transtornos mentais, deve-se chegar ao fundo, a causa, porque você pode fazer Reiki, pode fazer Auriculoterapia, pode fazer o melhor tratamento médico, mas se você não chega a causa, de nada vale. Tem que buscar uma solução para o problema que a pessoa tem. Nós precisamos da conversa com o paciente" (M2).

A extensão da assistência às famílias das pessoas em sofrimento mental foi mencionada como um aspecto que merece ser revisto pela equipe, conforme exemplifica-se no relato: "[...] uma coisa que a gente tem que olhar com outros olhos também, é a questão das famílias, do espaço que esse paciente convive" (E2).

Alguns participantes citaram a criação de espaços de lazer e locais para a prática de exercícios físicos, destinados à população, como estratégia para auxiliar na promoção e prevenção ao sofrimento mental. "Vamos pensar para frente, construir uma área de recreação para todos os grupos” (M2). "A academia de saúde, que antes tinha” (M3).

Outra sugestão que emergiu foi a realização de um processo de Educação Permanente em Saúde (EPS) para a equipe sobre a temática, conforme exemplifica-se no relato:

“Tudo a longo prazo, porque esta questão de saúde mental não dá para fazer na corrida, precisaria desse treinamento para os profissionais e também para os pacientes [...] nas escolas trabalhar muito isso com as crianças hoje, para o futuro" (E1).

"Eu penso que não deveria ficar aqui, que você deveria ir nas rádios, divulgar para as pessoas, que as pessoas saibam, porque não é assim, vem aqui pega uma medicação e vai embora, isso não é assim” (M1).

"Você deveria divulgar esse trabalho, porque a maioria da população não sabe dessa realidade" (E2).

\section{Discussão}

As medidas adotadas para conter o avanço da transmissão da COVID-19, como o uso de máscaras, o reforço das medidas de higiene e o distanciamento social, somados a insegurança de uma possível contaminação, a ausência de tratamentos, o luto pela perda de conhecidos e familiares acometidos pela doença, as questões socioeconômicas e a exposição constante a notícias sobre a pandemia acabaram impactando negativamente na saúde mental da população (Nabuco, 2020; Duarte \& et al., 2021). Diante desse cenário, a literatura pontua sobre a relevância da APS, bem como das UBS e do Núcleo Ampliado de Saúde da Família (NASF), enquanto serviços privilegiados para práticas de cuidado à população, principalmente em um momento de emergência pública, uma vez que estes serviços já costumam atender usuários em sofrimento mental (Duarte \& et al., 2021).

Neste contexto, identificou-se que as principais fragilidades citadas pelos participantes deste estudo no cuidado em saúde mental na APS, envolvem o uso de psicotrópicos. O primeiro aspecto citado pelos profissionais, diz respeito a não aceitação pelos usuários em sofrimento mental de terapêuticas não farmacológicas, como opções para tratar seu problema. A realidade evidenciada pelos profissionais vai ao encontro do que ocorre não só no Brasil, mas também nos países desenvolvidos, que apontam a preferência das pessoas em utilizar fármacos para o tratamento do sofrimento mental (Favero \& et al., 2019).

De acordo com a literatura, um dos fatores que pode ser associado à preferência do tratamento medicamentoso é a cultura dos usuários, que associam a solução de seus problemas de saúde a determinados produtos disponibilizados pelos serviços de saúde como, por exemplo, o medicamento. É como se o fármaco tivesse a propriedade natural de proteger a saúde, como se a utilização deste, por si só, fosse responsável por todo o processo de cuidado, quando na realidade é um recurso limitado (Favero \& et al., 2019). Compreende-se que o fácil acesso aos medicamentos, que em sua maioria, são disponibilizados gratuitamente pela farmácia da UBS e a praticidade de sua utilização, também possam ser fatores 
responsáveis pela não aceitação ou adesão dos usuários em sofrimento mental por outras terapêuticas oferecidas no serviço, como a psicoterapia.

Não se pode eximir também, a responsabilidade dos profissionais de saúde, sobre esta predileção aos tratamentos medicamentosos, pois estudos demonstram que apesar da proposta de reorientação do modelo de cuidado às pessoas em sofrimento mental, muitos serviços de saúde continuam focando suas ações na medicalização, como cuidado principal a esta demanda (Borges \& et al., 2015; Frosi \& Tesser, 2015).

Com relação ao uso desnecessário de psicotrópicos por determinados usuários, mencionada pelos participantes, salienta-se que infelizmente, os profissionais estão tendo que agir sobre um conjunto de sofrimentos, além da utilização de tratamentos medicamentosos em saúde mental a longo prazo pelas pessoas. Tal situação está sendo equiparada às abordagens consolidadas para doenças ou condições crônicas como hipertensão e diabetes, nas quais o cuidado fundamenta-se em uma conduta farmacológica permanente, os medicamentos de "uso contínuo" (Frosi \& Tesser, 2015).

Para os autores anteriormente citados, outro fator que pode ser atribuído ao uso desnecessário de psicotrópicos pode estar relacionado ao modelo de assistência que vem sendo prestado, ao longo dos anos, às pessoas em sofrimento mental na APS. Esta acontece, na maioria das vezes, de forma fragmentada, sem a realização de uma escuta qualificada ou da criação de vínculo com o usuário, resultando em inúmeras prescrições medicamentosas (Frosi \& Tesser, 2015).

No que diz respeito à prescrição inadequada de psicotrópicos, sem uma correta avaliação do indivíduo, salienta-se que tal prática vai contra o que é preconizado pelo Ministério da Saúde, que destaca ser importante a prescrição de doses mínimas necessárias para o tratamento dos transtornos mentais (Brasil, 2013).

A problemática do consumo de dois benzodiazepínicos diferentes, pelo mesmo usuário em um período inferior a um ano e a justificativa deste fato ser atribuída pelos participantes do estudo à falta de medicamentos na farmácia da UBS e à inexistência de um fluxo organizacional no serviço para atendimento às demandas de saúde mental, denota a importância da gestão no cuidado em saúde mental. Nesse sentido, o gestor deveria responsabilizar-se pela assistência farmacêutica no SUS, garantindo aos usuários acesso ao(s) medicamento(s), tendo na assistência farmacêutica uma ação de saúde efetiva e institucionalizada (Medeiros Filho \& et al., 2018).

Ainda discorrendo sobre as fragilidades relacionadas à utilização de psicotrópicos, evidenciou-se nesta pesquisa, a preocupação por parte dos profissionais sobre o consumo concomitante de dois ou mais psicofármacos pelos usuários atendidos. De acordo com estudo realizado a partir de prescrições de psicotrópicos, há uma alta frequência de interações medicamentosas associadas ao elevado número de fármacos em cada receita médica. Tal situação, denota a necessidade dos médicos repensarem a prescrição de vários medicamentos, a fim de reduzir a prática da polifarmácia nos serviços de saúde (Balen \& et al., 2017).

Apesar de, geralmente, ser o médico o responsável pelas orientações sobre o uso de psicofármacos aos usuários, considera-se importante a atuação de outros profissionais da equipe, como o enfermeiro, que pode orientar o usuário durante a consulta de enfermagem em relação a ocorrência de efeitos adversos com o uso conjunto de psicotrópicos, enfatizando a prevenção dos abusos e a promoção do uso racional, minimizando possíveis consequências desta prática (Balen \& et al., 2017; Borges \& et al., 2015). Neste sentido, pode-se compreender o uso racional de medicamentos, quando a pessoa recebe o fármaco apropriado à sua necessidade clínica, na dose e posologia correta, por um período de tempo adequado e ao menor custo para si e para a comunidade (OMS, 2002).

Para Borges e et al. (2015) é indiscutível o benefício da utilização de psicotrópicos no manejo dos transtornos mentais. Porém, é fundamental a avaliação dos riscos e benefícios desta terapêutica por parte dos médicos da APS. Considerase relevante também, que as pessoas sejam orientadas não só quanto ao melhor tratamento para seu caso, mas em relação à 
correta utilização desta categoria de fármacos, visto que o uso inadequado destes pode submeter os indivíduos há diversos riscos a sua saúde que podem ser graves.

Outro aspecto relevante que emergiu da análise dos dados foi o relato dos participantes sobre uma investigação, realizada pela equipe, a qual identificou que o principal motivo para que houvesse o retorno frequente dos mesmos usuários ao serviço eram questões relacionadas ao sofrimento mental. A falta de resolutividade dos problemas apresentados pelas pessoas, pode ser atribuída à inexistência de uma abordagem integral aos indivíduos no serviço. Pois, sabe-se que existe uma gama de situações em que os sofrimentos físico e mental apresentam-se de forma articulada, denotando que as doenças físicas e mentais compõem partes indissociáveis do ser humano (Frosi \& Tesser, 2015).

De acordo, com Flores (2020) as práticas no campo da saúde mental devem ir além do transtorno mental propriamente dito, e do foco exclusivo aos tratamentos, para que de fato ocorra o sucesso da terapêutica e cura dos agravos, bem como a superação das situações de vulnerabilidade e estigmatização. É necessário que haja uma cultura de cuidado e de suporte social aos indivíduos, que vise não somente a melhora do quadro, mas que abarque a troca de olhares e um contato pessoal próximo que possibilite mudanças de percepções e sentidos.

Souza e et al. (2017) mencionam que a Estratégia Saúde da Família (ESF), como parte da Rede de Atenção Psicossocial (RAPS) possui importante papel no cuidado em saúde mental da população assistida, uma vez, que está inserida no território onde as pessoas vivem. Este cenário contribui para que as equipes ofereçam cuidados integrais à saúde, bem como para a identificação precoce e a viabilidade de tratamentos, favorecendo ainda, a redução do estigma relacionado aos usuários com transtorno mental.

Os participantes relataram a importância da coordenação e longitudinalidade do cuidado. Nesse sentido, Mendes (2011) defende que o atributo coordenação tem uma importância maior nas Redes de Atenção à saúde (RAS), já que sem este os demais atributos, como o primeiro contato, a longitudinalidade e a integralidade seriam dificultadas. Outra pesquisa (Hirdes \& Scarparo, 2015; Hirdes \& Silva, 2017) evidenciou a importância da longitudinalidade, a necessidade de adoção de novas perspectivas epistemológicas; a incorporação da supervisão e apoio como uma ferramenta de trabalho em saúde mental na APS por meio do apoio matricial; o alargamento das competências disciplinares; a composição com equipes multidisciplinares na condução e cogestão das intervenções, o que remete a um trabalho eminentemente interdisciplinar.

Entende-se que todas as pessoas com queixas de sofrimento mental, deveriam ser acompanhadas pela equipe até a solução do problema apresentado. Apesar de um profissional participante da pesquisa ter mencionado sobre a impossibilidade de acompanhar todos os usuários, compreende-se que se houvesse uma organização adequada e o envolvimento dos diversos atores que compõem as equipes, seria possível a realização destes acompanhamentos a longo prazo. Neste sentido, vislumbrase que os Agentes Comunitários de Saúde (ACS) poderiam auxiliar neste trabalho.

Conforme evidenciado neste estudo, a família do usuário em sofrimento mental muitas vezes não é contemplada pelo cuidado ofertado pela equipe. Este fato demonstra outra fragilidade no que tange a assistência às demandas de saúde mental na APS, indo contra ao cuidado que é proposto às pessoas em sofrimento mental, que visa compreender não só o indivíduo, mas a sua singularidade e o contexto social no qual este está inserido, incluindo-se aqui a família. O vínculo entre os profissionais das ESF, a família e a comunidade é considerado como fundamental para que as ações da equipe tenham impacto positivo na saúde da população (Brasil, 2013).

Diante da preocupação com a saúde mental de crianças e adolescentes em vulnerabilidade social no município, devido as medidas que foram impostas para prevenção da COVID-19, salienta-se que a literatura aponta para a necessidade da realização de atividades de educação em saúde, com enfoque nas famílias, como formas de auxiliar no cuidado à saúde mental de crianças e adolescentes em tempos de pandemia por COVID-19. Tais ações podem se dar através de atendimentos no SUS, de atividades comunitárias ou por meios impressos, eletrônicos ou digitais, devendo ser adaptadas à realidade das famílias, 
principalmente daquelas com vulnerabilidade econômica e condições sanitárias inadequadas, como aquelas que residem em comunidades pobres e favelas (Almeida \& et al., 2020).

Discorrendo ainda sobre o cuidado a saúde mental em tempos de pandemia, compreende-se ser indispensável que os profissionais da APS desenvolvam estratégias de cuidado a todos indivíduos, independentemente de sua faixa etária, uma vez que a literatura destaca que, entre um terço e metade da população pode vir a apresentar alguma manifestação de sofrimento mental, caso não haja uma intervenção específica para as reações e sintomas manifestados neste período (Melo \& et al., 2020).

Frente às sugestões propostas pelos participantes para qualificar a assistência em saúde mental na APS, destaca-se que as mesmas vão ao encontro do que a literatura recomenda (Barros \& et al., 2019; Hirdes \& Scarparo, 2015, Hirdes \& Silva, 2017). Enfatiza-se que para o cuidado em saúde mental é necessário que os profissionais lancem mão de todos os instrumentos que possam facilitar o manejo dos casos, como a proximidade com as famílias, o matriciamento, a intersetorialidade, o trabalho em equipe, dentre outras (Barros \& et al., 2019).

Para a OMS (2002) as necessidades das pessoas em sofrimento mental e de suas famílias são múltiplas, variadas e diferem nas diversas fases das doenças, requerendo uma ampla variedade de serviços para proporcionar cuidados abrangentes. Neste sentido, destaca-se a importância das RAPS que possuem como objetivo criar, ampliar e articular os pontos de atenção à saúde para pessoas em sofrimento ou Transtorno Mental e seus familiares. Esta rede é constituída pela APS, atenção psicossocial especializada, atenção de urgência e emergência, atenção residencial transitória, atenção hospitalar, estratégias de desinstitucionalização e reabilitação psicossocial. Os pontos que constituem a RAPS na APS são a UBS, o NASF e os Centros de Convivência (Brasil, 2013). A utilização de grupos, atividades físicas, PICs, dentre outras, são estratégias importantes, que podem ser utilizadas pelas equipes para tratar o sofrimento físico e mental dos usuários, pois vão além da terapêutica medicamentosa, e são consideradas resolutivas (Frosi \& Tesser, 2015).

No que tange a sugestão do desenvolvimento de processos de EPS sobre a temática para a equipe, um estudo (Costa \& et al., 2017) demonstrou que estas atividades são fundamentais para que os profissionais estejam habilitados a atender as demandas de saúde mental, dentro da perspectiva da atenção psicossocial, sem reproduzir a assistência que era dispensada aos indivíduos dentro da lógica manicomial.

Frente a realização de atividades educativas voltadas à população sobre o uso racional de psicotrópicos, salienta-se que cabe aos profissionais da APS e farmacêuticos a elaboração de estratégias que possam identificar e prevenir os resultados negativos associados à medicação, promovendo o uso racional de medicamentos, principalmente através de práticas educativas (Melo \& Pauferro, 2020).

Considera-se fundamental o aprofundamento da temática tanto para a equipe, quanto para a população, contemplando principalmente ações voltadas à prevenção e promoção da saúde mental e o uso racional de psicotrópicos. Tais ações de educação em saúde devem contemplar também as crenças, percepções e atitudes dos usuários em relação às suas doenças, aos medicamentos e aos tratamentos, não bastando somente prestar recomendações para melhorar a saúde e prevenir as patologias. Mas dentro deste contexto, possibilitar o diálogo, a indagação, a reflexão, o questionamento e a ação compartilhada entre profissionais e população (Melo \& Pauferro, 2020).

\section{Considerações Finais}

Os resultados encontrados no presente estudo evidenciaram que as fragilidades no cuidado à saúde mental na APS estão relacionadas às questões envolvendo o uso de psicotrópicos, a inexistência de acompanhamento à família dos usuários em sofrimento mental e a ausência de um fluxo organizacional no serviço para o atendimento das demandas de saúde mental.

Existe uma preocupação por parte dos profissionais, acerca das consequências que as medidas impostas pela pandemia da COVID-19 podem acarretar na saúde mental de crianças e adolescentes em vulnerabilidade social. Tal situação, 
pode ser compreendida como uma lacuna no cuidado, que exige adoção urgente de medidas, por parte do gestor e da equipe multiprofissional. Com relação às propostas para qualificar a assistência em saúde mental na APS, foram citadas o cuidado com enfoque integral do usuário e sua família, a realização de processos de educação permanente sobre a temática para os profissionais, o desenvolvimento de ações voltadas à população sobre as questões de saúde mental, a implantação de espaços de lazer e atividade física no município e o desenvolvimento de ações de prevenção e promoção à saúde mental para crianças e adolescentes.

Pode-se concluir afirmando, que as fragilidades evidenciadas neste estudo demonstram a necessidade urgente de se reelaborar as estratégias de cuidado em saúde mental na APS, principalmente devido ao risco a que estão expostas algumas pessoas, com o uso inadequado de psicotrópicos. Compreende-se que o enfermeiro, ao possuir competências adequadas (conhecimentos, habilidades e atitudes), pode contribuir para a qualificação da assistência em saúde mental na APS. Para tanto, considera-se fundamental que as instituições formadoras dos profissionais de saúde, invistam no ensino em saúde mental de forma transversal, para atuação destes nos diversos contextos e níveis de assistência, como na APS.

As evidências deste estudo servem de base para a realização de futuras investigações acerca do cuidado em saúde mental na APS. Espera-se ainda, que contribuam para o desenvolvimento de um processo crítico-reflexivo por parte dos gestores e profissionais de saúde que atuam na APS, sobre a importância do aperfeiçoamento profissional no campo da saúde mental, para que de fato haja uma assistência qualificada e resolutiva aos usuários em sofrimento mental, principalmente neste período de pandemia por COVID-19.

Destacam-se como limitações deste estudo, a participação de apenas alguns membros da equipe da APS, sendo necessário abranger outros profissionais e também, o gestor do serviço. Entende-se que tais participações poderiam proporcionar melhor compreensão acerca da problemática envolvida, no que tange o cuidado aos usuários em sofrimento mental, bem como, uma sensibilização sobre a importância de uma mudança nas práticas de cuidado a esta demanda na APS. No entanto, salienta-se que estas limitações não comprometem a qualidade dos dados obtidos.

\section{Referências}

Almeida, S., Brito, R., Alves, M., Abranches, D., Wanderley, D., Rossano, C. \& Barros, R. (2020). COVID-19 pandemic: practical guide for promoting the mental he.alth of children and adolescents. Resid Pediatr. 10(2): 1-4. http://residenciapediatrica.com.br/sumario/50.

Ávila, B. M. \& Siniak, S. D. (2017) Ações de saúde mental desenvolvidas em uma estratégia saúde da família. Rev Enferm UFSM. 7(3): 388-397, https://periodicos.ufsm.br/reufsm/article/view/26237/pdf.

Balen, E., Giordani, F., Cano, F., Zonzini, T., Klein, A., Vieira, H. \& Mantovani, C. (2017). Interações medicamentosas potenciais entre medicamentos psicotrópicos dispensados. J Bras Psiquiatr. 66(3): 172-177. https://www.scielo.br/j/jbpsiq/a/ybdCdYkfjWLfgPRmPWyRwnx/abstract/?lang=pt.

Bardin, L. (2011). Análise de conteúdo. Edições 70.

Barros, S., Nóbrega, S. P. S M., Santos, C. J., Fonseca, M. L., \& Floriano, M. S. (2019). Saúde mental na atenção primária: processo saúde-doença, segundo profissionais de saúde. Rev Bras Enferm. 72(6): 1687-95. http://dx.doi.org/10.1590/0034-7167-2018-0743

Borges, T. L., Miasso, I. A., Vedana, G. G., Telles Filho, P. C. \& Hegadoren, M. K. (2015). Prevalence in the use of psychotropics and associated factors in primary health care. Acta Paul. Enferm. 28(4): 344-349. https://www.scielo.br/j/ape/a/CQCsPM6djQyyRhCcy3VhWHd/?lang=pt

Brasil. (2013). Cadernos de Atenção Básica, no 34. Saúde Mental. Secretaria de Atenção à Saúde, Departamento de Atenção Básica. http://bvsms.saude.gov.br/bvs/publicacoes/cadernos_atencao_basica_34_saude_mental.pdf

Brasil. (2012). Resolução $\mathrm{n}^{\circ}$ 466, de 12 de dezembro de 2012. Diário Oficial [da] República Federativa do Brasil. https://conselho.saude.gov.br/r esolucoes/2012/Reso466.pdf

Brasil. (2006). Política Nacional de Atenção Básica. Ministério da Saúde: Brasília. 60 p. http://bvsms.saude.gov.br/bvs/publicacoes/politica_ nacional_atencao_basica_2006.pdf

Campos, B. D.; Bezerra, C. I. \& Jorge, B. S. (2018). Mental health care technologies: Primary Care practices and processes. Rev. Bras Enf. 71(5): 2101-8. http://dx.doi.org/10.1590/0034-7167-2017-0478

Costa, D. T., Gonçalves, C. L., Manhães, P. S., Cortez, A. E. \& Tavares, M. (2017). Contribuindo para a educação permanente na saúde mental. POBS. 23(7): 9-15. https://ojs3.perspectivasonline.com.br/biologicas_e_saude/article/view/647/845 
COVID-19: Recomendações Gerais Fiocruz. https://www.fiocruzbrasilia.fiocruz.br/wp-content/uploads/2020/04/Sa\%C3\%BAde-Mental-eAten\%C3\%A7\%C3\%A3o-Psicossocial-na-Pandemia-Covid-19-recomenda\%C3\%A7\%C3\%B5es-gerais.pdf

Dias, B. J., Resende, F. M., Regne, G. R., Reinaldo, A. M., Pereira, M. O. \& Tavares, M. L. (2020). Cuidado em saúde mental e atenção primária em saúde como campo formador para a enfermagem. SMAD. Rev. Eletrônica Saúde Mental Álcool Drog. 16(2): 49-56. http://pepsic.bvsalud.org/scielo.php?script=sci_arttext\&pid=S1806-69762020000200007

Duarte, N., Jorge, B. S. M., Silva, F. M. D., Souza, C. B. D., Oliveira, S. R., Barroso, P. \& Lourinho, L. (2021). Estratégias de promoção da saúde mental na atenção primária à saúde no contexto da Covid-19: uma revisão integrativa. Research, Society and Development, 10(11): e176101119527. http://dx.doi.org/10.33448/rsd-v10i11.19527

Favero, C., Rodrigues, J., Silva, P., Santos, D., Barroso, A., Andrade, D., Barbosa, S. \& Stefanello, S. (2019). Grupo de gestão autônoma da medicação num centro de atenção psicossocial: experiência de usuários. Revista de Enfermagem Referência. https://www.redalyc.org/journal/3882/388260457009/388260457009.pdf.

Flores, D. (2020). A importância dos profissionais de saúde na desinstitucionalização do estigma dos sujeitos com transtornos mentais. Revista Portuguesa de Enfermagem de Saúde Mental 1(23): 41-46. https://scielo.pt/scielo.php?script=sci_arttext\&pid=S1647-21602020000100006?script=sci_arttext\&pid=S164721602020000100006

Frosi, R. V. \& Tesser, C. D. (2015). Práticas assistenciais em saúde mental na atenção primária à saúde: análise a partir de experiências desenvolvidas em Florianópolis, Brasil. Ciênc. Saúde Coletiva. 20(10): 3151-3161. https://www.scielo.br/j/csc/a/CXcZMLxNPRFLf8ys9qXRkLR/abstract/?lang=pt

Franco, T. B. \& Merhy, E. E. (2012) Cartografias do trabalho e cuidado em saúde. Rev Tempus Actas Saúde Coletiva, 6(2): 151-163. https://www.tempus.unb.br/index.php/tempus/issue/view/95

Hirdes, A., \& Scarparo, H. (2015). The maze and the minotaur: mental health in primary health care. Cien Saude Colet., 20(2): 383-93. https://www.scielo.br/j/csc/a/jk7v4HcTBCFjvj7vBXfFp6R/abstract/?lang=en

Hirdes, A., \& Silva, M. (2017). Articulation Between Matrix Support in Mental Health and Health Care Network. Psicol. Estud. 22(3): 383-394, https://periodicos.uem.br/ojs/index.php/PsicolEstud/article/view/32579

Machado, T. M., Carvalho, N. I., Brandão, M. S. \& Vilarinho, M. C. (2015). A Roda de Conversa como Planejamento de Ações: Relato de Experiência. Revista Eletrônica Gestão \&Saúde. 1(6): 751-761.https://periodicos.unb.br/index.php/rgs/article/view/2707.

Martini, L. (2020). Qual o papel da atenção primária no cuidado da saúde mental? Informa-SUS UFScar. https://www.informasus.ufscar.br/qual-o-papel-daatencao-primaria-no-cuidado-da-saude-mental/

Medeiros Filho, A., Azevedo, M., Pinto, R. \& Silva, S. (2018). Uso de psicofármacos na Atenção Primária à Saúde. Rev Bras Promoç Saúde, 31(3): 1-12. https://periodicos.unifor.br/RBPS/article/view/7670

Melo, R. C., \& Pauferro, V. R. (2020). Educação em saúde para a promoção do uso racional de medicamentos e as contribuições do farmacêutico neste contexto. Braz. J. of Develop., 6(5): 32162-32173. https://www.brazilianjournals.com/index.php/BRJD/article/view/10805

MELO, B. \& et al. (org). Saúde mental e atenção psicossocial na pandemia COVID-19: recomendações para gestores. Rio de Janeiro: Fiocruz, 2020. Cartilha. $13 \mathrm{p}$.

Mendes, E. V. (2011). As redes de atenção à saúde. Organização Pan-americana da Saúde; Brasília, (2a ed.),

Nishiura, H., Linton, N. \& Akhmetzhanov, A. (2020). Serial interval of novel coronavirus (COVID-19) infections. Int J Infect Dis 2020, 93(1): 284-286. https://www.ijidonline.com/article/S1201-9712(20)30119-3/fulltext

Nabuco, G., Oliveira, M. \& Afonso, M. (2020). O impacto da pandemia pela COVID-19 na saúde mental: qual é o papel da Atenção Primária à Saúde? Rev. Brasileira de Medicina de Família e Comunidade. 15(42): 1-11. https://rbmfc.org.br/rbmfc/article/view/2532

Organización Mundial de La Salud (OMS). (2002). Promoción del Uso Racional de Medicamentos: componentes centrales. Perspectivas políticas sobre medicamentos de la OMS. 5(1): 1-6. https://apps.who.int/iris/bitstream/handle/10665/67532/WHO_EDM_2002.3_spa.pdf;jsessionid=FF9E5949B 5BE9DAA0884BB3D8A9D4196? sequence=1.

Ornell, F., Schuch, J. B., Sordi, N. \& Kessler, F. (2020). Pandemia de medo e COVID-19: impactos na saúde mental e possíveis estratégias. Revista debates in psychiatry. Editorial. http://www.ufrgs.br/ufrgs/noticias/arquivos/pandemia-de-medo-e-covid-19-impacto-na-saude-mental-e-possiveis-estrategias.

Souza, J., Almeida, L., Luis, M., Nievas, A., Veloso, T., Barbosa, S. \& et al. (2017). Mental health in the Family Health Strategy as perceived by health professionals. Rev. bras. enferm. 70(5): 935-941. HTTP://www.scielo.br/pdf/reben/v70n5/0034-7167-reben-70-05-0935.pdf

Thiollent, M. (2011). Metodologia da pesquisa-ação. (18a ed.), Cortez. 